\title{
Article
}

\section{On the Generalized Laplace Transform}

\author{
Paul Bosch ${ }^{1,+} \oplus$, Héctor José Carmenate García ${ }^{2,+}$, José Manuel Rodríguez ${ }^{3,+} \oplus$ and José María Sigarreta ${ }^{2, *,+}+(\mathbb{)}$ \\ 1 Facultad de Ingeniería, Universidad del Desarrollo, Ave. La Plaza 680, San Carlos de Apoquindo, Las Condes, \\ Santiago 7550000, Chile; pbosch@udd.cl \\ 2 Centro Acapulco, Facultad de Matemática, Universidad Autónoma de Guerrero, Calle Chilpancingo, \\ Acapulco de Juárez, Guerrero 39610, Mexico; 20253352@uagro.mx \\ 3 Departamento de Matemáticas, Universidad Carlos III de Madrid, Avenida de la Universidad 30, Leganés, \\ 28911 Madrid, Spain; jomaro@math.uc3m.es \\ * Correspondence: 14366@uagro.mx \\ + The authors contributed equally to this work.
}

Citation: Bosch, P.; Carmenate García, H.J.; Rodríguez, J.M.; Sigarreta, J.M. On the Generalized Laplace Transform. Symmetry 2021, 13, 669. https://doi.org/10.3390/ sym 13040669

Academic Editor: Carlo Cattani

Received: 18 March 2021

Accepted: 4 April 2021

Published: 13 April 2021

Publisher's Note: MDPI stays neutral with regard to jurisdictional claims in published maps and institutional affiliations.

Copyright: (c) 2021 by the authors. Licensee MDPI, Basel, Switzerland. This article is an open access article distributed under the terms and conditions of the Creative Commons Attribution (CC BY) license (https:// creativecommons.org/licenses/by/ $4.0 /)$.

\begin{abstract}
In this paper we introduce a generalized Laplace transform in order to work with a very general fractional derivative, and we obtain the properties of this new transform. We also include the corresponding convolution and inverse formula. In particular, the definition of convolution for this generalized Laplace transform improves previous results. Additionally, we deal with the generalized harmonic oscillator equation, showing that this transform and its properties allow one to solve fractional differential equations.
\end{abstract}

Keywords: fractional derivative; convolution; generalized Laplace transform

\section{Introduction}

Differential and integral calculus provide numerous tools for solving modeled problems, but there are many phenomena whose formulations are far more precise if fractional calculus is used. Fractional calculus is as old as calculus itself, and extends derivation and integration to arbitrary non-integer orders.

Liouville gave two definitions of derivative [1], treating the fractional order derivative as an integral, albeit with certain limitations. Anton Karl Grünwald, in 1867 [2], and Aleksey Vasilievich Létnikov, in 1868 [3], proposed a new definition of fractional derivative based on the definition of iterated derivative, known as the Grünwald-Létnikov differo-integral operator. Later, in 1898, the definition given by Liouville was improved by Riemann in a posthumously published manuscript [4]. In 1969, Michele Caputo gave a new definition that allowed the physical interpretation of many problems, since it has ordinary initial conditions unlike the derivative of Riemann, so it is usually used in application problems [5].

The concept of the conformable fractional derivative was introduced in [6]; then [7-12] proposed derivatives of local character, which opened up a new horizon in fractional calculus.

Fractional calculus is now successfully used in a wide range of models in physics, economics and biology. Of particular importance are the physical applications in the theory of viscoelasticity, in the study of anomalous diffusion phenomena and in electromagnetic theory. There is currently a growing interest in other very different fields, such as circuit theory and the physics of the atmosphere. Additionally, among economists, the use of fractional calculus concepts is increasing. There are well-known fractional models such as that of the change of heat load intensity on the walls of a furnace, the Bagley-Torvik equation, the neural fractional order model, the deformation law and the model of the spread of Dengue fever, where the advantage of using a non-integer formulation of the derivative is evident [13-29]. 
As far as classical calculus is concerned, ordinary and partial differential equations describe how certain quantities vary in time, such as the current in an electric circuit, the oscillations in a vibrating membrane or the heat flow through an insulated conductor. These equations usually have initial conditions that describe the state of the system at time $t=0$. The Laplace transform method solves most of these problems, by transforming the original equation into an elementary algebraic expression, which can then be transformed back into the solution of the original problem [30,31]. The Laplace transform was useful used in the study of fractional differential equations; see, e.g., [29,32,33].

Therefore, it is interesting to extend the Laplace transform to differential equations with non-integer orders, for the generalized fractional derivative exposed in [12]. Furthermore, earlier works dealing with the Laplace transform for fractional derivatives introduced some convolutions that have some drawbacks. We introduce here a new concept of convolution that avoids the problems of the previous definitions; in particular, this convolution uses a symmetric operator; see Theorem 8 . We also include the corresponding inverse formula in Theorem 9. Finally, we solve the generalized harmonic oscillator as an exemplary application to show the reliability of the generalized Laplace transform method:

$$
G_{T}^{\alpha}\left(G_{T}^{\alpha} f\right)(t)+c^{2} f(t)=u(t),
$$

where $G_{T}^{\alpha} f(t)$ is the conformable fractional derivative operator of order $\alpha \in(0,1]$, and $c$ is a constant.

\section{Preliminaries}

Let us recall the definition of a local generalized fractional derivative in [12].

Given $s \in \mathbb{R}$, we denote by $\lceil s\rceil$ the upper integer part of $s$, i.e., the smallest integer greater than or equal to $s$.

Definition 1. Given an interval $I \subseteq \mathbb{R}, f: I \rightarrow \mathbb{R}, \alpha \in \mathbb{R}^{+}$and a positive continuous function $T(t, \alpha)$ on I for each $\alpha$, the derivative $G_{T}^{\alpha} f$ of $f$ of order $\alpha$ at the point $t \in I$ is defined by

$$
G_{T}^{\alpha} f(t)=\lim _{h \rightarrow 0} \frac{1}{h^{\lceil\alpha\rceil}} \sum_{k=0}^{\lceil\alpha\rceil}(-1)^{k}\left(\begin{array}{c}
\lceil\alpha\rceil \\
k
\end{array}\right) f(t-k h T(t, \alpha)) .
$$

If $a=\min \{t \in I\}$ (respectively, $b=\max \{t \in I\}$ ), then $G_{T}^{\alpha} f(a)$ (respectively, $G_{T}^{\alpha} f(b)$ ) is defined with $h \rightarrow 0^{-}$(respectively, $h \rightarrow 0^{+}$) instead of $h \rightarrow 0$ in the limit.

If we choose the function $T(t, \alpha)=t^{\lceil\alpha\rceil-\alpha}$, then we obtain the following particular case of $G_{T}^{\alpha}$, defined in [6]. Note that $T(t, \alpha)=t^{[\alpha\rceil-\alpha}=1$ for every $\alpha \in \mathbb{N}$.

Definition 2. Let I be an interval $I \subseteq(0, \infty), f: I \rightarrow \mathbb{R}$ and $\alpha \in \mathbb{R}^{+}$. The conformable derivative $G^{\alpha} f$ of $f$ of order $\alpha$ at the point $t \in I$ is defined by

$$
G^{\alpha} f(t)=\lim _{h \rightarrow 0} \frac{1}{h^{\lceil\alpha\rceil}} \sum_{k=0}^{\lceil\alpha\rceil}(-1)^{k}\left(\begin{array}{c}
\lceil\alpha\rceil \\
k
\end{array}\right) f\left(t-k h t^{\lceil\alpha\rceil-\alpha}\right) .
$$

We know from the classical calculus that if $f$ is a function defined in a neighborhood of the point $t$, and there exists $D^{n} f(t)$, then

$$
D^{n} f(t)=\lim _{h \rightarrow 0} \frac{1}{h^{n}} \sum_{k=0}^{n}(-1)^{k}\left(\begin{array}{l}
n \\
k
\end{array}\right) f(t-k h) .
$$

Therefore, if $\alpha=n \in \mathbb{N}$ and $f$ is smooth enough, then Definition 2 coincides with the classical definition of the $n$-th derivative.

In [6], a conformable derivative is defined in the following way. 
Definition 3. Given $f:(0, \infty) \rightarrow \mathbb{R}$ and $\alpha \in(0,1]$, the derivative of $f$ of order $\alpha$ at the point $t$ is defined by

$$
T_{\alpha} f(t)=\lim _{h \rightarrow 0} \frac{f(t)-f\left(t-h t^{1-\alpha}\right)}{h} .
$$

It is clear then that $T_{\alpha}$ is a particular case of $G^{\alpha}$ when $\alpha \in(0,1]$ and $T(t, \alpha)=t^{1-\alpha}$. See $[9,34,35]$ for more information on $T_{\alpha}$.

The following results in [12] contain some basic properties of the derivative $G_{T}^{\alpha}$.

Lemma 1. Let $I$ be an interval $I \subseteq \mathbb{R}, f: I \rightarrow \mathbb{R}$ and $\alpha \in \mathbb{R}^{+}$.

(1) If there exists $D^{\lceil\alpha\rceil} f$ at the point $t \in I$, then $f$ is $G_{T}^{\alpha}$-differentiable at $t$ and $G_{T}^{\alpha} f(t)=$ $T(t, \alpha)^{\lceil\alpha\rceil} D^{\lceil\alpha\rceil} f(t)$.

(2) If $\alpha \in(0,1]$, then $f$ is $G_{T}^{\alpha}$-differentiable at $t \in I$ if and only if $f$ is differentiable at $t$; in this case, we have $G_{T}^{\alpha} f(t)=T(t, \alpha) f^{\prime}(t)$.

Lemma 2. Let $I$ be an interval $I \subseteq \mathbb{R}, f, g: I \rightarrow \mathbb{R}$ and $\alpha \in \mathbb{R}^{+}$. Assume that $f, g$ are $G_{T}^{\alpha}$-differentiable functions at $t \in I$. Then the following statements hold:

(1) $a f+b g$ is $G_{T}^{\alpha}$-differentiable at $t$ for every $a, b \in \mathbb{R}$, and $G_{T}^{\alpha}(a f+b g)(t)=a G_{T}^{\alpha} f(t)+$ $b G_{T}^{\alpha} g(t)$.

(2) If $\alpha \in(0,1]$, then $f g$ is $G_{T}^{\alpha}$-differentiable at $t$ and $G_{T}^{\alpha}(f g)(t)=f(t) G_{T}^{\alpha} g(t)+g(t) G_{T}^{\alpha} f(t)$.

(3) If $\alpha \in(0,1]$ and $g(t) \neq 0$, then $f / g$ is $G_{T}^{\alpha}$-differentiable at $t$ and $G_{T}^{\alpha}\left(\frac{f}{g}\right)(t)=$ $\frac{g(t) G_{T}^{\alpha} f(t)-f(t) G_{T}^{\alpha} g(t)}{g(t)^{2}}$.

(4) $G_{T}^{\alpha}(\lambda)=0$, for every $\lambda \in \mathbb{R}$.

(5) $\quad G_{T}^{\alpha}\left(t^{p}\right)=\frac{\Gamma(p+1)}{\Gamma(p-\lceil\alpha\rceil+1)} t^{p-\lceil\alpha\rceil} T(t, \alpha)^{\lceil\alpha\rceil}$, for every $p \in \mathbb{R} \backslash \mathbb{Z}^{-}$.

(6) $G_{T}^{\alpha}\left(t^{-n}\right)=(-1)^{\lceil\alpha\rceil} \frac{\Gamma(n+\lceil\alpha\rceil)}{\Gamma(n)} t^{-n-\lceil\alpha\rceil} T(t, \alpha)^{\lceil\alpha\rceil}$, for every $n \in \mathbb{Z}^{+}$.

Lemma 3. Let $\alpha \in(0,1], g$ be a $G_{T}^{\alpha}$-differentiable function at $t$ and $f$ be a differentiable function at $g(t)$. Then $f \circ g$ is $G_{T}^{\alpha}$-differentiable at $t$, and $G_{T}^{\alpha}(f \circ g)(t)=f^{\prime}(g(t)) G_{T}^{\alpha} g(t)$.

\section{On the Generalized Laplace Transform}

In this section, we assume that the function $T$ is positive and continuous on $(0, \infty)$, and satisfies for some $\varepsilon>0$

$$
\int_{0}^{\varepsilon} \frac{d \omega}{T(\omega, \alpha)}<\infty, \quad \text { and } \quad \int_{0}^{\infty} \frac{d \omega}{T(\omega, \alpha)}=\infty,
$$

for each $0<\alpha \leq 1$.

Let us define for each $0<\alpha \leq 1$ and $c, t \in \mathbb{R}$

$$
E_{\alpha}(c, t)=\exp \left(c \int_{0}^{t} \frac{d \omega}{T(\omega, \alpha)}\right) .
$$

Note that $E_{\alpha}(c, t)$ is an eigenfunction for the operator $G_{T}^{\alpha}$, since

$$
\begin{aligned}
G_{T}^{\alpha}\left(E_{\alpha}(c, t)\right) & =T(t, \alpha)\left(\exp \left(c \int_{0}^{t} \frac{d \omega}{T(\omega, \alpha)}\right)\right)^{\prime} \\
& =T(t, \alpha) \exp \left(c \int_{0}^{t} \frac{d \omega}{T(\omega, \alpha)}\right) \frac{c}{T(t, \alpha)}=c E_{\alpha}(c, t) .
\end{aligned}
$$

Thus,

$$
\left(E_{\alpha}(c, t)\right)^{\prime}=c E_{\alpha}(c, t) \frac{1}{T(t, \alpha)}
$$


Given $0<\alpha \leq 1$ and a measurable function $f:[0, \infty) \rightarrow \mathbb{R}$, we define its generalized Laplace transform as

$$
\mathcal{L}_{T}^{\alpha}[f](s)=\int_{0}^{\infty} E_{\alpha}(-s, t) f(t) \frac{d t}{T(t, \alpha)},
$$

if $\mathcal{L}_{T}^{\alpha}[|f|](s)<\infty$, i.e., $E_{\alpha}(-s, t) f(t) / T(t, \alpha) \in L^{1}([0, \infty))$. If we consider complex-valued functions instead of real-valued functions, then we can obtain similar results.

The following properties of the generalized Laplace transform are elementary.

Proposition 1. Let $c, k \in \mathbb{R}, 0<\alpha \leq 1$ and $f, g:[0, \infty) \rightarrow \mathbb{R}$ be functions such that there exist $\mathcal{L}_{T}^{\alpha}[f](s)$ and $\mathcal{L}_{T}^{\alpha}[g](s)$ for some s.

(1) Then

$$
\mathcal{L}_{T}^{\alpha}[c f+k g](s)=c \mathcal{L}_{T}^{\alpha}[f](s)+k \mathcal{L}_{T}^{\alpha}[g](s) .
$$

(2) If there exists $\mathcal{L}_{T}^{\alpha}\left[E_{\alpha}(c, t) f(t)\right](s)$, then

$$
\mathcal{L}_{T}^{\alpha}\left[E_{\alpha}(c, t) f(t)\right](s)=\mathcal{L}_{T}^{\alpha}[f](s-c) .
$$

The following results summarize the main properties of the generalized Laplace transform.

Theorem 1. Let $f:[0, \infty) \rightarrow \mathbb{R}$ be a function such that there exists $\mathcal{L}_{T}^{\alpha}[f](s)$ for some s and $0<\alpha \leq 1$. Then

$$
\mathcal{L}_{T}^{\alpha}[f(t)](s)=L[f(u(x))](s),
$$

where $L$ denotes the usual Laplace transform, and $u(x)$ is the inverse function of

$$
x(t)=\int_{0}^{t} \frac{d \omega}{T(\omega, \alpha)} .
$$

Proof. Since $T(t, \alpha)$ is a positive continuous function on $(0, \infty)$, and it satisfies for some $\varepsilon>0$

$$
\int_{0}^{\varepsilon} \frac{d \omega}{T(\omega, \alpha)}<\infty, \quad \int_{0}^{\infty} \frac{d \omega}{T(\omega, \alpha)}=\infty,
$$

the function $x:[0, \infty) \rightarrow[0, \infty)$ given by

$$
x(t)=\int_{0}^{t} \frac{d \omega}{T(\omega, \alpha)}
$$

is continuous and strictly increasing, $x(0)=0$ and $\lim _{t \rightarrow \infty} x(t)=\infty$. Thus, $x(t)$ is a homeomorphism on $[0, \infty)$ and so is its inverse function.

The change of variable

$$
x=\int_{0}^{t} \frac{d \omega}{T(\omega, \alpha)}, \quad d x=\frac{d t}{T(t, \alpha)}, \quad t=u(x),
$$

allows one to obtain

$$
\begin{aligned}
\mathcal{L}_{T}^{\alpha}[f](s) & =\int_{0}^{\infty} E_{\alpha}(-s, t) f(t) \frac{d t}{T(t, \alpha)}=\int_{0}^{\infty} e^{-s x(t)} f(t) \frac{d t}{T(t, \alpha)} \\
& =\int_{0}^{\infty} e^{-s x} f(u(x)) d x=L[f(u(x))](s) .
\end{aligned}
$$


Corollary 1. Let $f:[0, \infty) \rightarrow \mathbb{R}$ be a function such that there exists $L[f](s)$ for some s and $0<\alpha \leq 1$. Then there exists the generalized Laplace transform at $s$ of

$$
f\left(\int_{0}^{t} \frac{d \omega}{T(\omega, \alpha)}\right)
$$

and

$$
\mathcal{L}_{T}^{\alpha}\left[f\left(\int_{0}^{t} \frac{d \omega}{T(\omega, \alpha)}\right)\right](s)=L[f](s) .
$$

Corollary 1 has the following consequence.

Proposition 2. There exists the generalized Laplace transform of the following functions for $0<\alpha \leq 1$ :

(1) If $s>0$, then

$$
\mathcal{L}_{T}^{\alpha}[1](s)=\frac{1}{s}
$$

(2) If $a \in \mathbb{R}$ and $s>a$, then

$$
\mathcal{L}_{T}^{\alpha}\left[E_{\alpha}(a, t)\right](s)=\frac{1}{s-a} .
$$

(3) If $b>-1$ and $s>0$, then

$$
\mathcal{L}_{T}^{\alpha}\left[\left(\int_{0}^{t} \frac{d \omega}{T(\omega, \alpha)}\right)^{b}\right](s)=\frac{\Gamma(b+1)}{s^{b+1}} .
$$

(4) If $b>0$ and $s>0$, then

$$
\mathcal{L}_{T}^{\alpha}\left[\sin \left(b \int_{0}^{t} \frac{d \omega}{T(\omega, \alpha)}\right)\right](s)=\frac{b}{s^{2}+b^{2}} .
$$

(5) If $b>0$ and $s>0$, then

$$
\mathcal{L}_{T}^{\alpha}\left[\cos \left(b \int_{0}^{t} \frac{d \omega}{T(\omega, \alpha)}\right)\right](s)=\frac{s}{s^{2}+b^{2}} .
$$

(6) If $a \in \mathbb{R}, b>-1$ and $s>0$, then

$$
\mathcal{L}_{T}^{\alpha}\left[E_{\alpha}(a, t)\left(\int_{0}^{t} \frac{d \omega}{T(\omega, \alpha)}\right)^{b}\right](s)=\frac{\Gamma(b+1)}{(s-a)^{b+1}} .
$$

(7) If $a \in \mathbb{R}, b>0$ and $s>a$, then

$$
\mathcal{L}_{T}^{\alpha}\left[E_{\alpha}(a, t) \sin \left(b \int_{0}^{t} \frac{d \omega}{T(\omega, \alpha)}\right)\right](s)=\frac{b}{(s-a)^{2}+b^{2}} .
$$

(8) If $a \in \mathbb{R}, b>0$ and $s>a$, then

$$
\mathcal{L}_{T}^{\alpha}\left[E_{\alpha}(a, t) \cos \left(b \int_{0}^{t} \frac{d \omega}{T(\omega, \alpha)}\right)\right](s)=\frac{s-a}{(s-a)^{2}+b^{2}} .
$$

(9) If $a>0$ and $s>a$, then

$$
\mathcal{L}_{T}^{\alpha}\left[\sinh \left(a \int_{0}^{t} \frac{d \omega}{T(\omega, \alpha)}\right)\right](s)=\frac{a}{s^{2}-a^{2}} .
$$


(10) If $a>0$ and $s>a$, then

$$
\mathcal{L}_{T}^{\alpha}\left[\cosh \left(a \int_{0}^{t} \frac{d \omega}{T(\omega, \alpha)}\right)\right](s)=\frac{s}{s^{2}-a^{2}} .
$$

The following result gives a sufficient condition for the existence of the generalized Laplace transform.

Theorem 2. Let $f:[0, \infty) \rightarrow \mathbb{R}$ be a measurable function and $0<\alpha \leq 1$. If there exist constants $k>0$ and $c \in \mathbb{R}$ such that

$$
|f(t)| \leq k E_{\alpha}(c, t)
$$

for every $t>0$, then there exists $\mathcal{L}_{T}^{\alpha}[f](s)$ for every $s>c$ and

$$
\left|\mathcal{L}_{T}^{\alpha}[f](s)\right| \leq \frac{k}{s-c} .
$$

Proof. If $s>c$, then Proposition 2 gives

$$
\begin{aligned}
\left|\mathcal{L}_{T}^{\alpha}[f](s)\right| & \leq \int_{0}^{\infty}\left|\frac{E_{\alpha}(-s, t) f(t)}{T(t, \alpha)}\right| d t \leq k \int_{0}^{\infty} \frac{E_{\alpha}(-s, t) E_{\alpha}(c, t)}{T(t, \alpha)} d t \\
& =k \mathcal{L}_{T}^{\alpha}\left[E_{\alpha}(c, t)\right](s)=\frac{k}{s-c}
\end{aligned}
$$

and there exists $\mathcal{L}_{T}^{\alpha}[f](s)$.

The following result shows that $\mathcal{L}_{T}^{\alpha}$ is the appropriate Laplace transform in order to work with the fractional derivative $G_{T}^{\alpha}$.

Theorem 3. Let $f:[0, \infty) \rightarrow \mathbb{R}$ be a locally absolutely continuous function such that there exist $\mathcal{L}_{T}^{\alpha}[f](s)$ and $\mathcal{L}_{T}^{\alpha}\left[G_{T}^{\alpha} f\right](s)$ for some s and $0<\alpha \leq 1$. Then

$$
\mathcal{L}_{T}^{\alpha}\left[G_{T}^{\alpha} f\right](s)=s \mathcal{L}_{T}^{\alpha}[f](s)-f(0) .
$$

Proof. If we apply integration by parts to the integral

$$
\mathcal{L}_{T}^{\alpha}\left[G_{T}^{\alpha} f\right](s)=\int_{0}^{\infty} E_{\alpha}(-s, t) G_{T}^{\alpha} f(t) \frac{d t}{T(t, \alpha)}=\int_{0}^{\infty} E_{\alpha}(-s, t) f^{\prime}(t) d t
$$

with

$$
\begin{array}{ll}
u=E_{\alpha}(-s, t), & d u=-s E_{\alpha}(-s, t) \frac{d t}{T(t, \alpha)}, \\
d v=f^{\prime}(t) d t, & v=f(t),
\end{array}
$$

we obtain

$$
\begin{aligned}
\mathcal{L}_{T}^{\alpha}\left[G_{T}^{\alpha} f\right](s) & =\left[E_{\alpha}(-s, t) f(t)\right]_{t=0}^{t=\infty}+s \int_{0}^{\infty} E_{\alpha}(-s, t) f(t) \frac{d t}{T(t, \alpha)} \\
& =\lim _{t \rightarrow \infty} E_{\alpha}(-s, t) f(t)-f(0)+s \mathcal{L}_{T}^{\alpha}[f](s) .
\end{aligned}
$$

Note that there exists $\lim _{t \rightarrow \infty} E_{\alpha}(-s, t) f(t)$ and it is finite, since there exist $\mathcal{L}_{T}^{\alpha}[f](s)$ and $\mathcal{L}_{T}^{\alpha}\left[G_{T}^{\alpha} f\right](s)$.

When seeking a contradiction, assume that $\lim _{t \rightarrow \infty} E_{\alpha}(-s, t) f(t)=k \neq 0$. Thus,

$$
E_{\alpha}(-s, t) f(t) \frac{1}{T(t, \alpha)} \approx \frac{k}{T(t, \alpha)}
$$


as $t \rightarrow \infty$. Since

$$
\int^{\infty} \frac{d \omega}{T(\omega, \alpha)}=\infty \Rightarrow E_{\alpha}(-s, t) f(t) \frac{1}{T(t, \alpha)} \notin L^{1}([0, \infty))
$$

and $\mathcal{L}_{T}^{\alpha}[f](s)$ does not exist, there is a contradiction. Hence, $\lim _{t \rightarrow \infty} E_{\alpha}(-s, t) f(t)=0$ and the conclusion holds.

We can iterate this formula.

Theorem 4. Let $f:[0, \infty) \rightarrow \mathbb{R}$ be a $C^{1}$ function such that $f^{\prime}$ is a locally absolutely continuous function and there exist $\mathcal{L}_{T}^{\alpha}[f](s), \mathcal{L}_{T}^{\alpha}\left[G_{T}^{\alpha} f\right](s)$ and $\mathcal{L}_{T}^{\alpha}\left[G_{T}^{\alpha}\left(G_{T}^{\alpha} f\right)\right](s)$ for some s and $0<\alpha \leq 1$. Then

$$
\begin{aligned}
\mathcal{L}_{T}^{\alpha}\left[G_{T}^{\alpha}\left(G_{T}^{\alpha} f\right)\right](s) & =s^{2} \mathcal{L}_{T}^{\alpha}[f](s)-s f(0)-G_{T}^{\alpha} f(0) \\
& =s^{2} \mathcal{L}_{T}^{\alpha}[f](s)-s f(0)-T(0, \alpha) f^{\prime}(0) .
\end{aligned}
$$

Proof. Theorem 3 and Lemma 1 give

$$
\begin{aligned}
\mathcal{L}_{T}^{\alpha}\left[G_{T}^{\alpha} f\right](s) & =s \mathcal{L}_{T}^{\alpha}[f](s)-f(0), \\
\mathcal{L}_{T}^{\alpha}\left[G_{T}^{\alpha}\left(G_{T}^{\alpha} f\right)\right](s) & =s \mathcal{L}_{T}^{\alpha}\left[G_{T}^{\alpha} f\right](s)-G_{T}^{\alpha} f(0)=s \mathcal{L}_{T}^{\alpha}\left[G_{T}^{\alpha} f\right](s)-T(0, \alpha) f^{\prime}(0),
\end{aligned}
$$

and these equalities give the conclusion.

Theorem 5 below shows that the following integral operator plays an important role in our study.

$$
J_{T}^{\alpha}(f)(t)=\int_{0}^{t} \frac{f(\omega)}{T(\omega, \alpha)} d \omega
$$

Theorem 5. Let $f:[0, \infty) \rightarrow \mathbb{R}$ be a function such that there exist $\mathcal{L}_{T}^{\alpha}[f](s)$ and $\mathcal{L}_{T}^{\alpha}\left[J_{T}^{\alpha}(f)\right](s)$ for some $s$ and $0<\alpha \leq 1$. Then

$$
\mathcal{L}_{T}^{\alpha}\left[J_{T}^{\alpha}(f)\right](s)=\frac{1}{s} \mathcal{L}_{T}^{\alpha}[f](s) .
$$

Proof. Since there exists $\mathcal{L}_{T}^{\alpha}[f](s)$, we have that there exists $J_{T}^{\alpha}(f)(t)$ for every $t>0$. Additionally, $J_{T}^{\alpha}(f)(t)$ is locally absolutely continuous on $[0, \infty)$ and

$$
\left(J_{T}^{\alpha}(f)\right)^{\prime}(t)=\frac{f(t)}{T(t, \alpha)}, \quad G_{T}^{\alpha}\left(J_{T}^{\alpha}(f)\right)(t)=f(t)
$$

for almost every $t \in[0, \infty)$. Thus, Theorem 3 applied to the function $J_{T}^{\alpha}(f)$ gives

$$
\mathcal{L}_{T}^{\alpha}[f](s)=\mathcal{L}_{T}^{\alpha}\left[G_{T}^{\alpha}\left(J_{T}^{\alpha}(f)\right)\right](s)=s \mathcal{L}_{T}^{\alpha}\left[J_{T}^{\alpha}(f)\right](s)-J_{T}^{\alpha}(f)(0)=s \mathcal{L}_{T}^{\alpha}\left[J_{T}^{\alpha}(f)\right](s) .
$$

Let us prove some regularity properties of the generalized Laplace transform.

Theorem 6. Let $f:[0, \infty) \rightarrow \mathbb{R}$ be a function such that there exists $\mathcal{L}_{T}^{\alpha}[f]\left(s_{0}\right)$ for some $s_{0} \in \mathbb{R}$, and $0<\alpha \leq 1$. Then:

(1) There exists $\mathcal{L}_{T}^{\alpha}[f](s)$ for every $s \in \mathbb{C}$ with real part $\operatorname{Re} s \geq s_{0}$.

(2) $\mathcal{L}_{T}^{\alpha}[f](s)$ is continuous on the closed halfplane $\left\{s \in \mathbb{C}: \operatorname{Re} s \geq s_{0}\right\}$.

(3) $\mathcal{L}_{T}^{\alpha}[f](s)$ is analytic on the open halfplane $H\left(s_{0}\right)=\left\{s \in \mathbb{C}: \operatorname{Re} s>s_{0}\right\}$.

(4) If $s \in H\left(s_{0}\right)$ and $n$ is a positive integer, then

$$
\frac{d^{n} \mathcal{L}_{T}^{\alpha}[f]}{d s^{n}}(s)=(-1)^{n} \int_{0}^{\infty} E_{\alpha}(-s, t) f(t)\left(\int_{0}^{t} \frac{d \omega}{T(\omega, \alpha)}\right)^{n} \frac{d t}{T(t, \alpha)}
$$


Proof. If $\operatorname{Re} s \geq s_{0}$, then

$$
\left|\frac{E_{\alpha}(-s, t) f(t)}{T(t, \alpha)}\right|=\frac{E_{\alpha}(-\operatorname{Res}, t)|f(t)|}{T(t, \alpha)} \leq \frac{E_{\alpha}\left(-s_{0}, t\right)|f(t)|}{T(t, \alpha)} .
$$

Since $E_{\alpha}\left(-s_{0}, t\right) f(t) / T(t, \alpha) \in L^{1}([0, \infty))$, we conclude that $E_{\alpha}(-s, t) f(t) / T(t, \alpha) \in$ $L^{1}([0, \infty))$, and so, there exists $\mathcal{L}_{T}^{\alpha}[f](s)$.

Since (4) holds for every $s$ with $\operatorname{Re} s \geq s_{0}$ and $E_{\alpha}\left(-s_{0}, t\right) f(t) / T(t, \alpha)$ is an integrable function on $[0, \infty)$ which does not depend on $s$, the dominated convergence theorem gives that $\mathcal{L}_{T}^{\alpha}[f](s)$ is a continuous function on this closed halfplane.

Let $\gamma:[a, b] \rightarrow \mathbb{C}$ be a closed curve contained in the open halfplane $H\left(s_{0}\right)$. Fubini's theorem gives

$$
\begin{aligned}
\int_{\gamma} \mathcal{L}_{T}^{\alpha}[f](s) d s & =\int_{\gamma} \int_{0}^{\infty} E_{\alpha}(-s, t) f(t) \frac{d t}{T(t, \alpha)} d s \\
& =\int_{0}^{\infty} \int_{\gamma} E_{\alpha}(-s, t) d s f(t) \frac{d t}{T(t, \alpha)} .
\end{aligned}
$$

Since $E_{\alpha}(-s, t)$ is an analytic function in the variable $s$ for each fixed $t$, Cauchy's theorem gives $\int_{\gamma} E_{\alpha}(-s, t) d s=0$ and so,

$$
\int_{\gamma} \mathcal{L}_{T}^{\alpha}[f](s) d s=\int_{0}^{\infty} \int_{\gamma} E_{\alpha}(-s, t) d s f(t) \frac{d t}{T(t, \alpha)}=\int_{0}^{\infty} 0 \cdot f(t) \frac{d t}{T(t, \alpha)}=0 .
$$

Since $\gamma$ is an arbitrary closed curve contained in the open halfplane $H\left(s_{0}\right)$, Morera's theorem gives that $\mathcal{L}_{T}^{\alpha}[f](s)$ is analytic on $H\left(s_{0}\right)$.

Fix $s_{1}>s_{0}$ and a positive integer $n$. If $s \in H\left(s_{1}\right)$, then

$$
\begin{aligned}
\mid \frac{E_{\alpha}(-s, t) f(t)}{T(t, \alpha)} & \left(\int_{0}^{t} \frac{d \omega}{T(\omega, \alpha)}\right)^{n} \mid=\frac{E_{\alpha}(-\operatorname{Re} s, t)|f(t)|}{T(t, \alpha)}\left(\int_{0}^{t} \frac{d \omega}{T(\omega, \alpha)}\right)^{n} \\
& \leq \frac{E_{\alpha}\left(-s_{1}, t\right)|f(t)|}{T(t, \alpha)}\left(\int_{0}^{t} \frac{d \omega}{T(\omega, \alpha)}\right)^{n} \\
& =\frac{E_{\alpha}\left(-s_{0}, t\right)|f(t)|}{T(t, \alpha)} E_{\alpha}\left(-\left(s_{1}-s_{0}\right), t\right)\left(\int_{0}^{t} \frac{d \omega}{T(\omega, \alpha)}\right)^{n} .
\end{aligned}
$$

It is well-known that there exists a positive constant $c_{n}$ such that $e^{-x} \leq c_{n} x^{-n}$ for every $x \geq 1$. As in Theorem 1, denote by $u(x)$ the inverse function of

$$
x(t)=\int_{0}^{t} \frac{d \omega}{T(\omega, \alpha)} .
$$

If we define

$$
t_{0}=u\left(\frac{1}{s_{1}-s_{0}}\right)
$$

and $t \geq t_{0}$, then

$$
\begin{aligned}
\int_{0}^{t} \frac{d \omega}{T(\omega, \alpha)}=x(t) \geq x\left(t_{0}\right) & =\frac{1}{s_{1}-s_{0}} \Rightarrow\left(s_{1}-s_{0}\right) \int_{0}^{t} \frac{d \omega}{T(\omega, \alpha)} \geq 1 \\
\Rightarrow \quad E_{\alpha}\left(-\left(s_{1}-s_{0}\right), t\right) & =\exp \left(-\left(s_{1}-s_{0}\right) \int_{0}^{t} \frac{d \omega}{T(\omega, \alpha)}\right) \\
\leq & \frac{c_{n}}{\left(s_{1}-s_{0}\right)^{n}}\left(\int_{0}^{t} \frac{d \omega}{T(\omega, \alpha)}\right)^{-n} .
\end{aligned}
$$


Thus, we obtain

$$
\begin{aligned}
\left|\frac{E_{\alpha}(-s, t) f(t)}{T(t, \alpha)}\left(\int_{0}^{t} \frac{d \omega}{T(\omega, \alpha)}\right)^{n}\right| & \leq \frac{E_{\alpha}\left(-s_{0}, t\right)|f(t)|}{T(t, \alpha)} E_{\alpha}\left(-\left(s_{1}-s_{0}\right), t\right)\left(\int_{0}^{t} \frac{d \omega}{T(\omega, \alpha)}\right)^{n} \\
& \leq \frac{c_{n}}{\left(s_{1}-s_{0}\right)^{n}} \frac{E_{\alpha}\left(-s_{0}, t\right)|f(t)|}{T(t, \alpha)}
\end{aligned}
$$

for every $t \geq t_{0}$. Additionally, we have for every $0 \leq t \leq t_{0}$

$$
\left|\frac{E_{\alpha}(-s, t) f(t)}{T(t, \alpha)}\left(\int_{0}^{t} \frac{d \omega}{T(\omega, \alpha)}\right)^{n}\right| \leq\left(\int_{0}^{t_{0}} \frac{d \omega}{T(\omega, \alpha)}\right)^{n} \frac{E_{\alpha}\left(-s_{0}, t\right)|f(t)|}{T(t, \alpha)} .
$$

If we define

$$
M=\max \left\{\frac{c_{n}}{\left(s_{1}-s_{0}\right)^{n}},\left(\int_{0}^{t_{0}} \frac{d \omega}{T(\omega, \alpha)}\right)^{n}\right\}
$$

then

$$
\left|\frac{E_{\alpha}(-s, t) f(t)}{T(t, \alpha)}\left(\int_{0}^{t} \frac{d \omega}{T(\omega, \alpha)}\right)^{n}\right| \leq M \frac{E_{\alpha}\left(-s_{0}, t\right)|f(t)|}{T(t, \alpha)}
$$

for every $t \geq 0$.

Since $E_{\alpha}\left(-s_{0}, t\right) f(t) / T(t, \alpha) \in L^{1}([0, \infty))$, the function

$$
\frac{E_{\alpha}(-s, t) f(t)}{T(t, \alpha)}\left(\int_{0}^{t} \frac{d \omega}{T(\omega, \alpha)}\right)^{n}
$$

is bounded by an integrable function on $[0, \infty)$ which does not depend on $s \in H\left(s_{1}\right)$. Therefore, the dominated convergence theorem gives that

$$
\begin{aligned}
\frac{d^{n} \mathcal{L}_{T}^{\alpha}[f]}{d s^{n}}(s) & =\frac{d^{n}}{d s^{n}}\left(\int_{0}^{\infty} E_{\alpha}(-s, t) f(t) \frac{d t}{T(t, \alpha)}\right) \\
& =\int_{0}^{\infty} \frac{\partial^{n}}{\partial s^{n}}\left(E_{\alpha}(-s, t)\right) f(t) \frac{d t}{T(t, \alpha)} \\
& =(-1)^{n} \int_{0}^{\infty} E_{\alpha}(-s, t) f(t)\left(\int_{0}^{t} \frac{d \omega}{T(\omega, \alpha)}\right)^{n} \frac{d t}{T(t, \alpha)} .
\end{aligned}
$$

Since this formula holds for $s \in H\left(s_{1}\right)$ and every $s_{1}>s_{0}$, it holds for every $s \in$ $H\left(s_{0}\right)$.

Theorem 7. Let $f:[0, \infty) \rightarrow \mathbb{R}$ be a function such that there exists $\mathcal{L}_{T}^{\alpha}[f]\left(s_{0}\right)$ for some $s_{0} \in \mathbb{R}$ and $0<\alpha \leq 1$. Then

$$
\lim _{s \rightarrow \infty} \mathcal{L}_{T}^{\alpha}[f](s)=0 .
$$

Proof. Since there exists $\mathcal{L}_{T}^{\alpha}[f]\left(s_{0}\right)$, we have

$$
\int_{0}^{1} \frac{|f(t)|}{T(t, \alpha)} d t<\infty
$$

Thus, for each $\varepsilon>0$ there exists $\delta>0$ with

$$
\int_{0}^{\delta} \frac{|f(t)|}{T(t, \alpha)} d t<\frac{\varepsilon}{2}
$$

If $s>s_{0}$, then

$$
\left|\frac{E_{\alpha}(-s, t) f(t)}{T(t, \alpha)}\right|=\frac{E_{\alpha}\left(-s_{0}, t\right)|f(t)|}{T(t, \alpha)} E_{\alpha}\left(-\left(s-s_{0}\right), t\right) .
$$


Hence

$$
\begin{aligned}
\left|\mathcal{L}_{T}^{\alpha}[f](s)\right| & \leq \int_{0}^{\delta} \frac{E_{\alpha}(-s, t)|f(t)|}{T(t, \alpha)} d t+\int_{\delta}^{\infty} \frac{E_{\alpha}(-s, t)|f(t)|}{T(t, \alpha)} d t \\
& <\frac{\varepsilon}{2}+\int_{\delta}^{\infty} \frac{E_{\alpha}\left(-s_{0}, t\right)|f(t)|}{T(t, \alpha)} E_{\alpha}\left(-\left(s-s_{0}\right), t\right) d t \\
& \leq \frac{\varepsilon}{2}+\int_{\delta}^{\infty} \frac{E_{\alpha}\left(-s_{0}, t\right)|f(t)|}{T(t, \alpha)} E_{\alpha}\left(-\left(s-s_{0}\right), \delta\right) d t \\
& =\frac{\varepsilon}{2}+\mathcal{L}_{T}^{\alpha}[|f|]\left(s_{0}\right) E_{\alpha}\left(-\left(s-s_{0}\right), \delta\right) .
\end{aligned}
$$

Since $\lim _{s \rightarrow \infty} E_{\alpha}\left(-\left(s-s_{0}\right), \delta\right)=0$, there exists $N$ with

$$
\mathcal{L}_{T}^{\alpha}[|f|]\left(s_{0}\right) E_{\alpha}\left(-\left(s-s_{0}\right), \delta\right)<\varepsilon / 2
$$

for $s>N$. Thus, $\left|\mathcal{L}_{T}^{\alpha}[f](s)\right|<\varepsilon$ for $s>N$ and $\lim _{s \rightarrow \infty} \mathcal{L}_{T}^{\alpha}[f](s)=0$.

Let $f, g:[0, \infty) \rightarrow \mathbb{R}$ be measurable functions and $0<\alpha \leq 1$. Recall that we have defined

$$
x(t)=\int_{0}^{t} \frac{d \omega}{T(\omega, \alpha)}
$$

and $u(x)$ as the inverse function of $x(t)$. Let us define the generalized convolution of $f$ and $g$ as

$$
(f * g)(t)=\int_{0}^{t} f(u(x(t)-x(\omega))) g(\omega) \frac{d \omega}{T(\omega, \alpha)} .
$$

If $T(t, \alpha)=t^{1-\alpha}$, then

$$
x(t)=\int_{0}^{t} \frac{d \omega}{T(\omega, \alpha)}=\int_{0}^{t} \omega^{\alpha-1} d \omega=\frac{1}{\alpha} t^{\alpha}
$$

and $u(x)=(\alpha x)^{1 / \alpha}$. Thus, we have in this case

$$
\begin{aligned}
(f * g)(t) & =\int_{0}^{t} f(u(x(t)-x(\omega))) g(\omega) \frac{d \omega}{T(\omega, \alpha)} \\
& =\int_{0}^{t} f\left(\left(t^{\alpha}-\omega^{\alpha}\right)^{1 / \alpha}\right) g(\omega) \omega^{\alpha-1} d \omega .
\end{aligned}
$$

Our definition of convolution has the advantage that it is symmetric, unlike the one in [32] (Theorem 3). Another symmetric convolution is defined in [33] (Theorem 3.8), but this result contains a mistake (in the proof of Theorem 3.8 it is shown that the Laplace transform of the convolution is the product of two functions which are not the Laplace transform of the factors, since the integrals involve the functions $\phi\left(\xi^{\omega} / \omega\right)$ and $\psi\left(v^{\omega} / \omega\right)$ instead of $\phi(\xi)$ and $\psi(v)$, respectively).

Theorem 8. Let $f, g:[0, \infty) \rightarrow \mathbb{R}$ be functions such that there exist $\mathcal{L}_{T}^{\alpha}[f](s)$ and $\mathcal{L}_{T}^{\alpha}[g](s)$ for some $s$ and $0<\alpha \leq 1$. Then

$$
\mathcal{L}_{T}^{\alpha}[f * g](s)=\mathcal{L}_{T}^{\alpha}[f](s) \mathcal{L}_{T}^{\alpha}[g](s)
$$


Proof. By applying Fubini's theorem, we obtain

$$
\begin{aligned}
\mathcal{L}_{T}^{\alpha}[f & * g](s)=\int_{0}^{\infty} e^{-s x(t)}(f * g)(t) \frac{d t}{T(t, \alpha)}=\int_{0}^{\infty} e^{-s x(t)}(f * g)(t) x^{\prime}(t) d t \\
& =\int_{0}^{\infty} e^{-s x(t)} \int_{0}^{t} f(u(x(t)-x(\omega))) g(\omega) x^{\prime}(\omega) d \omega x^{\prime}(t) d t \\
& =\int_{0}^{\infty} \int_{\omega}^{\infty} e^{-s x(\omega)} e^{-s(x(t)-x(\omega))} f(u(x(t)-x(\omega))) g(\omega) x^{\prime}(t) d t x^{\prime}(\omega) d \omega .
\end{aligned}
$$

The change of variable $x(z)=x(t)-x(\omega), x^{\prime}(z) d z=x^{\prime}(t) d t$, gives

$$
\begin{aligned}
\mathcal{L}_{T}^{\alpha}[f & * g](s)=\int_{0}^{\infty} \int_{0}^{\infty} e^{-s x(\omega)} e^{-s x(z)} f(u(x(z))) g(\omega) x^{\prime}(z) d z x^{\prime}(\omega) d \omega \\
& =\int_{0}^{\infty} e^{-s x(z)} f(z) x^{\prime}(z) d z \int_{0}^{\infty} e^{-s x(\omega)} g(\omega) x^{\prime}(\omega) d \omega \\
& =\mathcal{L}_{T}^{\alpha}[f](s) \mathcal{L}_{T}^{\alpha}[g](s) .
\end{aligned}
$$

\section{A Mellin's Inverse-Type Formula}

The classical Mellin inverse formula gives

$$
f(t)=L^{-1}(L[f](s))(t)=\frac{1}{2 \pi i} \lim _{T \rightarrow \infty} \int_{a-i T}^{a+i T} e^{s t} L[f](s) d s,
$$

where $L$ denotes the usual Laplace transform, and the integration is done along the vertical line $\{\Re s=a\}$ if this line is contained in the region of convergence of $L[f](s)$.

We present here a Mellin inverse-type formula for the generalized Laplace transform:

Theorem 9. Let $f:[0, \infty) \rightarrow \mathbb{R}$ be a function such that there exists $\mathcal{L}_{T}^{\alpha}[f](s)$ for some $s$ and $0<\alpha \leq 1$. Then

$$
f(t)=\frac{1}{2 \pi i} \lim _{T \rightarrow \infty} \int_{a-i T}^{a+i T} E_{\alpha}(s, t) \mathcal{L}_{T}^{\alpha}[f](s) d s,
$$

where the integration is done along the vertical line $\{\Re s=a\}$ if this line is contained in the region of convergence of $\mathcal{L}_{T}^{\alpha}[f](s)$.

Proof. Theorem 1 gives that

$$
\mathcal{L}_{T}^{\alpha}[f(t)](s)=L[f(u(x))](s),
$$

where $u(x)$ is the inverse function of

$$
x(t)=\int_{0}^{t} \frac{d \omega}{T(\omega, \alpha)} .
$$

Mellin's inverse formula gives

$$
f(u(t))=\frac{1}{2 \pi i} \lim _{T \rightarrow \infty} \int_{a-i T}^{a+i T} e^{s t} L[f(u(x))](s) d s=\frac{1}{2 \pi i} \lim _{T \rightarrow \infty} \int_{a-i T}^{a+i T} e^{s t} \mathcal{L}_{T}^{\alpha}[f](s) d s,
$$

where the integration is done along the vertical line $\{\Re s=a\}$ if this line is contained in the region of convergence of $\mathcal{L}_{T}^{\alpha}[f](s)$. Hence, 


$$
\begin{aligned}
f(t) & =f(u(x(t)))=\frac{1}{2 \pi i} \lim _{T \rightarrow \infty} \int_{a-i T}^{a+i T} e^{s x(t)} \mathcal{L}_{T}^{\alpha}[f](s) d s \\
& =\frac{1}{2 \pi i} \lim _{T \rightarrow \infty} \int_{a-i T}^{a+i T} E_{\alpha}(s, t) \mathcal{L}_{T}^{\alpha}[f](s) d s .
\end{aligned}
$$

\section{Generalized Harmonic Oscillator}

We want to study the equation of the generalized harmonic oscillator:

$$
G_{T}^{\alpha}\left(G_{T}^{\alpha} f\right)(t)+c^{2} f(t)=u(t), \quad f(0)=a, \quad G_{T}^{\alpha} f(0)=b,
$$

with $a, b, c \in \mathbb{R}, c \neq 0$.

By applying the generalized Laplace transform to this equation and using the fact that it is a linear operator, we have:

$$
\left\{\begin{array}{l}
\mathcal{L}_{T}^{\alpha}\left[G_{T}^{\alpha}\left(G_{T}^{\alpha}\right) f(t)\right](s)+c^{2} \mathcal{L}_{T}^{\alpha}[f(t)](s)=\mathcal{L}_{T}^{\alpha}[u(t)](s) \\
\quad f(0)=a, \quad G_{T}^{\alpha} f(0)=b
\end{array}\right.
$$

Using Theorem 4, this equation transforms into

$$
\begin{gathered}
s^{2} \mathcal{L}_{T}^{\alpha}[f(t)](s)-s f(0)-G_{T}^{\alpha} f(0)+c^{2} \mathcal{L}_{T}^{\alpha}[f(t)](s)=\mathcal{L}_{T}^{\alpha}[u(t)](s), \\
s^{2} \mathcal{L}_{T}^{\alpha}[f(t)](s)-a s-b+c^{2} \mathcal{L}_{T}^{\alpha}[f(t)](s)=\mathcal{L}_{T}^{\alpha}[u(t)](s), \\
\mathcal{L}_{T}^{\alpha}[f(t)](s)=\frac{a s+b}{s^{2}+c^{2}}+\mathcal{L}_{T}^{\alpha}[u(t)](s) \frac{1}{s^{2}+c^{2}} .
\end{gathered}
$$

Finally, Proposition 2 and Theorem 8 give

$$
f(t)=a \cos \left(c \int_{0}^{t} \frac{d \omega}{T(\omega, \alpha)}\right)+\frac{b}{c} \sin \left(c \int_{0}^{t} \frac{d \omega}{T(\omega, \alpha)}\right)+\frac{1}{c} u * \sin \left(c \int_{0}^{t} \frac{d \omega}{T(\omega, \alpha)}\right) .
$$

\section{Conclusions}

In this paper, we developed the theory of a generalized Laplace transform on fractional differential equations with a generalized fractional derivative $G_{T}^{\alpha}$, and we prove its properties. We also included the corresponding convolution and inverse formula. In particular, our definition of convolution for this Laplace transform has the advantage that it is symmetric, unlike the one in [32] (Theorem 3); another symmetric convolution is defined in [33] (Theorem 3.8), but this result contains a mistake (in the proof of Theorem 3.8 it is shown that the Laplace transform of the convolution is the product of two functions which are not the Laplace transform of the factors, since the integrals involve the functions $\phi\left(\xi^{\omega} / \omega\right)$ and $\psi\left(v^{\omega} / \omega\right)$ instead of $\phi(\xi)$ and $\psi(v)$, respectively). Additionally, we dealt with the generalized harmonic oscillator equation, showing that this generalized Laplace transform and its properties allow one to solve fractional differential equations.

Author Contributions: Investigation, P.B., H.J.C.G., J.M.R. and J.M.S. All authors contributed equally to the work. All authors have read and agreed to the published version of the manuscript.

Funding: This research received no external funding.

Institutional Review Board Statement: Not applicable.

Informed Consent Statement: Not applicable.

Data Availability Statement: Not applicable.

Acknowledgments: We would like to thank the referees for their comments, which have improved the paper. The research of José M. Rodríguez and José M. Sigarreta was supported by a grant from Agencia Estatal de Investigación (PID2019-106433GB-I00/AEI/10.13039/501100011033), 
Spain. The research of José M. Rodríguez is supported by the Madrid Government (Comunidad de Madrid-Spain) under the Multiannual Agreement with UC3M in the line of Excellence of University Professors (EPUC3M23), and in the context of the V PRICIT (Regional Programme of Research and Technological Innovation).

Conflicts of Interest: The authors declare no conflict of interest.

\section{References}

1. Liouville, J. Mémoire sur quelques questions de géométrie et de mécanique, et sur un nouveau genre de calcul pour résoudre ces questions. J. L'éCole Polytech. 1832, 13, 1-69.

2. Grünwald, A. Über "begrenzte" Derivation und deren Anwendung. Zangew. Math. Phys. 1867, 12, 441-480.

3. Letnikov, A.V. Theory of differentiation of an arbitrary order. Mat. Sb. 1868, 3, 1-68.

4. Riemann, B. Oeuvres Mathématiques de Riemann, 1st ed.; Gauthier-Villars et fils: Paris, France, 1898.

5. Caputo, M. Elasticitá e Dissipazione, 1st ed.; Zanichelli: Bologna, Italy, 1969.

6. Khalil, R.; Al Horani, M.; Yousef, A.; Sababheh, M. A new definition of fractional derivative. J. Comput. Appl. Math. 2014, 264, 65-70. [CrossRef]

7. Karci, A. Chain rule for fractional order derivatives. Sci. Innov. 2015, 3, 63-67. [CrossRef]

8. Almeida, R.; Guzowska, M.; Odzijewicz, T. A remark on local fractional calculus and ordinary derivatives. Open Math. 2016, 14, 1122-1124. [CrossRef]

9. Katugampola, V.N. A new fractional derivative with classical properties. J. Math. Anal. Appl. 2014, 6, 1-15.

10. Vanterler da C Sousa, J.; Capelas de Oliveira, E. A new truncated M-fractional derivative unifying some fractional derivatives with classical properties. Int. J. Anal. Appl. 2018, 16, 83-96.

11. Guzman, P.; Langton, G.; Motta, P.; Lugo, L.; Medina, J.; Valdes, N. A new definition of a fractional derivative of local type. J. Math. Anal. 2018, 9, 88-98.

12. Fleitas, A.; Nápoles Valdés, J.E.; Rodríguez, J.M.; Sigarreta, J.M. Note on the generalized conformable derivative. Rev. Unión Mat. Argent. 2015, in press. (stage of publication)

13. Oldham, K.; Spanier, J. The Fractional Calculus, Theory and Applications of Differentiation and Integration of Arbitrary Order, 1st ed.; Academic Press: New York, NY, USA, 1974.

14. Miller, K. An Introduction to Fractional Calculus and Fractional Differential Equations, 1st ed.; John Wiley and Sons: New York, NY, USA, 1993.

15. Podlubny, I. Fractional Differential Equations, 1st ed.; Academic Press: New York, NY, USA, 1999.

16. Kilbas, A.; Srivastava, H.; Trujillo, J. Fractional Differential Equations; Elsevier: New York, NY, USA, 2006; Volume 204.

17. Gómez, J. Analytical and Numerical solutions of a nonlinear alcoholism model via variable-order fractional differential equations. Phys. A Stat. Mech. Appl. 2018, 494, 52-75. [CrossRef]

18. Fleitas, A.; Gómez, J.; Nápoles, J.; Rodríguez, J.M.; Sigarreta, J.M. Analysis of the local Drude model involving the generalized fractional derivative. Opt. Int. J. Light Electron. Opt. 2019, 193, 163008. [CrossRef]

19. Anastasio, T. The fractional-order dynamics of brainstem vestibulo-oculomotor neurons. Biol. Cybern. 1994, 72, 69-79. [CrossRef]

20. Gerasimov, A.N. A generalization of linear laws of deformation and its application to problems of internal friction. Akad. Nauk SSSR. Prikl. Mat. Meh. 1948, 12, 251-260.

21. Torvik, P.; Bagley, R. On the appearance of the fractional derivative in the behavior of real materials. J. Appl. Mech. 1984, 51, 294-298. [CrossRef]

22. Hammad, M.A.; Khalil, R. Abel's formula and wronskian for comformable fractional differential equation. Int. J. Differ. Equ. Appl. 2014, 13, 177-183.

23. Atangana, A.; Baleanu, D.; Alsaedi, A. New properties of conformable derivative. Open Math. 2015, 13, 889-898. [CrossRef]

24. Baleanu, D.; Asad, J.H.; Jajarmi, A. The fractional model of spring pendulum: New features within different kernels. Proc. Rom. Acad. Ser. A 2018, 19, 447-454.

25. Hammad, M.A.; Khalil, R. Total fractional differentials with applications to exact fractional differential equations. Int. J. Comput. Math. 2018, 95, 1444-1452.

26. Baleanu, D.; Jajarmi, A.; Asad, J.H. Classical and fractional aspects of two coupled pendulums. Rom. Rep. Phys. 2019, 71, 103.

27. Baleanu, D.; Sadat, S.; Jajarmi, A.; Asad, J.H. New features of the fractional Euler-Lagrange equations for a physical system within non-singular derivative operator. Eur. Phys. J. Plus 2019, 134, 181. [CrossRef]

28. Bosch, P.; Gómez-Aguilar, J.F.; Rodríguez, J.M.; Sigarreta, J.M. Analysis of Dengue fever outbreak by generalized fractional derivative. Fractals 2020, 28, 12. [CrossRef]

29. Wei, Y.; Chen, Y.; Wang, Y.; Chen, Y.Q. Some Fundamental Properties on the Sampling Free Nabla Laplace Transform. In Proceedings of the 15th IEEE/ASME International Conference on Mechatronic and Embedded Systems and Applications, Anaheim, CA, USA, 18-21 August 2019.

30. Schiff, J. The Laplace Transform: Theory and Applications, 1st ed.; Springer: New York, NY, USA, 1999.

31. Widder, D. Laplace Transform, 6th ed.; Princeton University Press: Princeton, NJ, USA, 2015.

32. Eroglu, B.I.; Avci, D.; Ozdemir, N. Optimal control problem for a conformable fractional heat conduction equation. Acta Phys. Pol. A 2017, 132, 658-662. [CrossRef] 
33. Khan, N.A.; Razzaq, O.A.; Ayaz, M. Some properties and applications of conformable fractional laplace transform. J. Fract. Calc. Appl. 2018, 9, 72-81.

34. Abdejjawad, T. On conformable fractional calculus. J. Comput. Appl. Math. 2015, 279, 57-66. [CrossRef]

35. Jarad, F.; Ugurlu, E.; Abdeljawad, T.; Baleanu, D. On a new class of fractional operators. Adv. Differ. Equ. 2017, $247,1-16$. [CrossRef] 